\title{
ÍNDICE DE CERTIFICAÇÃO \\ DA SUSTENTABILIDADE EM CIDADES
}

\begin{abstract}
RESUMO
Desenvolver um Índice de Certificação de Cidades com base na modelagem urbana, capaz de demonstrar diferenciações quantitativas e qualitativas em termos da eficiência energética, com vistas à sustentabilidade social, econômica e ambiental em cidades, é o objetivo da presente pesquisa. Considera-se que a área de abrangência do setor urbano; a distribuição dos caminhos alternativos do sistema de vias; a densidade e compacidade edilícia; e, a economia de insumos naturais e antrópicos, são medidas de desempenho que aprimoram significativamente os parâmetros de análise das certificações atualmente utilizadas. Em específico, estas medidas de desempenho abarcam variáveis socioespaciais e técnico-ambientais compostas por propriedades técnicas, construtivas, morfológicas, sociais, políticas, econômicas, culturais, espaciais e ambientais, as quais constituem a materialidade fundamental para a vida em cidades. Neste sentido, faz-se a análise conceitual e teórica em torno dos principais processos e instrumentos de avaliação de sustentabilidade reconhecidos nacional e internacionalmente, de modo a embasar a metodologia para o desenvolvimento de cada medida de desempenho que compõe o Índice de Certificação de Cidades. Em relação aos resultados obtidos, trata-se de um instrumento que demonstra ser operacionalmente viável e promissor, apresentando resultados com alto grau de confiabilidade, servindo de parâmetro capaz de embasar decisões de gestão urbana em termos de planejamento, desenho e projeto urbano.
\end{abstract}

PALAVRAS-CHAVE: Planejamento urbano. Sustentabilidade urbana. Certificação de cidades

\section{CERTIFICATION INDEX OF SUSTAINABILITY IN CITIES}

\section{ABSTRACT}

Develop a Certification Cities Index based on urban modeling, able to demonstrate quantitative and qualitative differences in terms of energy efficiency, with view to social, economic and environmental sustainability in cities, is the goal of this research. It is considered that the coverage area of the urban sector; alternative ways of distributing the routes system; buildings density and compactness; and the economy of natural and anthropic inputs, are performance measures that significantly enhance the

\footnotetext{
1 Doutor em Planejamento Urbano e Regional - UFRGS; Arquiteto e Urbanista - UNISINOS, Professor do Curso de Graduação e Pós-Graduação em Arquitetura e Urbanismo - UNISINOS; Pesquisador CNPQ/CAPES. e-mail: silandre@unisinos.br
} 
analysis parameters of certifications currently used. In particular, these performance measures encompass socio-spatial variables, technical and environmental composed of technical, constructive, morphological, social, political, economic, cultural, spatial and environmental properties, which constitute the fundamental materiality for life in cities. In this sense, it is the conceptual and theoretical analysis around main processes and sustainability assessment tools national and internationally recognized, in order to base the methodology for the development of each performance measure that makes up the Certification Cities Index. Regarding the results obtained, it is an instrument that proves to be operationally viable and promising, showing results with a high degree of reliability, serving as a parameter able to base urban management decisions in terms of planning and urban design.

KEYWORDS: Urban planning. Urban sustainability . Cities Certification

\title{
ÍNDICE DE CERTIFICACIÓN DE SOSTENIBILIDAD EN LAS CIUDADES
}

\begin{abstract}
RESUMEN
Desarrollar uno Índice de Certificación de las Ciudades basado en modelado urbano, capaz de demostrar las diferencias cuantitativas y cualitativas en términos de eficiencia energética, con miras a la sostenibilidad social, económica y ambiental de las ciudades, es el objetivo de esta investigación. Se considera que el área de cobertura del sector urbano; formas alternativas de distribución del sistema de rutas; densidad edilicia y la compacidad; y la economía de los insumos naturales y artificiales, son medidas de rendimiento que mejoran significativamente los parámetros de análisis de las certificaciones que se utilizan actualmente. En particular, estas medidas de rendimiento abarcan variables socio-espacial, técnicas y ambientales compuestas de propiedades técnicas, constructivas, morfológicas, sociales, políticos, económicos, culturales, espaciales y ambientales, que constituyen la materialidad fundamental para la vida en las ciudades. En este sentido, es el análisis conceptual y teórico en torno a los procesos clave y herramientas de evaluación de la sostenibilidad reconocidos a nivel nacional e internacional, con el fin de fundamentar la metodología para el desarrollo de cada medida de desempeño que conforma el Índice de Certificación de las Ciudades. En cuanto a los resultados obtenidos, es un instrumento que resulta ser operativamente viable y prometedor, que muestra los resultados con un alto grado de fidelidad, que sirve como un parámetro capaz de fundamentar las decisiones de gestión urbana en términos de planificación y diseño urbano.
\end{abstract}

PALABRAS CLAVE : Planificación urbana . Sostenibilidad urbana . Certificación de la Ciudades

\section{INTRODUÇÃO E CONTEXTUALIZAÇÃO}

O tema certificação ${ }^{2}$ da sustentabilidade de setores urbanos relaciona-se à ideia de novos instrumentos para auxiliar no monitoramento permanente da qualidade de vida nas cidades projetadas, em processo de consolidação ou consolidadas. O termo sustentabilidade aplicado na presente pesquisa ${ }^{3}$, remete a setores urbanos ambientalmente corretos, socialmente responsáveis e

\footnotetext{
${ }^{2}$ Certificação consiste na emissão de um parecer, baseado numa decisão tomada após análise crítica, de que o atendimento aos requisitos especificados foi demonstrado e comprovado.

${ }_{3}^{3}$ Pesquisa apoiada pelo CNPQ - MCTI/CNPq/MEC/CAPES No 43/2013. Processo nº 409093/2013-0.
} 
economicamente viáveis. Enquanto que setores urbanos correspondem à delimitação de áreas da cidade em diferentes níveis, tais como o físico, o social, o econômico dentre outros, em razão da necessidade de intervenções e análises específicas.

Para aplicar o conceito de planejamento e gestão urbana sustentável faz-se necessário estabelecer índices, indicadores e/ou modelos urbanos, capazes de mensurar o desempenho de setores urbanos em termos da sustentabilidade social, econômica e ambiental. Neste sentido, o problema central está em distinguir quais propriedades são relevantes na consideração da certificação de cidades brasileiras. Parte-se da hipótese de que propriedades centradas em variáveis socioespaciais e técnico-ambientais apresentam maior abrangência e capacidade explicativa, cuja consideração em conjunto aprimora significativamente os critérios de análise das certificações atualmente utilizadas. O objetivo central da presente pesquisa é propor um modelo de sustentabilidade urbana, composto por indicadores centrados em medidas de desempenho, cujo índice seja parâmetro de análise de setores da cidade. Isto porque, reflexões sobre o desempenho técnico, construtivo, morfológico, social, político, econômico, cultural, espacial e ambiental - enquanto materialidade fundamental para a sustentabilidade - expressam a necessidade de conhecer melhor esse fenômeno urbano, de modo a subsidiar decisões de gestão, planejamento, desenho e projeto urbano com vistas à sustentabilidade social, econômica e ambiental das cidades.

\section{ANÁLISE CONJUGADA DE INDICADORES, ÍNDICES E MODELOS URBANOS}

Atualmente, a análise de determinadas variáveis, relações e efeitos complexos que os tecidos urbanos exercem nas pessoas constitui o desafio de pesquisa de diversos autores no âmbito dos indicadores, índices e modelos urbanos. Estes estudos, tanto em nível nacional quanto internacional, aplicam diferentes conceitos, teorias e métodos. O propósito destes é servir como meio para explicações não-determinísticas e absolutas de causa e efeito dos fatos observados, 
mas enfocar as diferentes possibilidades de compreensão das relações por detrás do fenômeno urbano.

Indicadores são parâmetros selecionados e considerados isoladamente ou combinados entre si de um determinado aspecto da realidade observada. A proposição do desenvolvimento de indicadores para avaliar a sustentabilidade surgiu na Conferência Mundial sobre o Meio Ambiente - Rio 92:

É preciso elaborar indicadores de desenvolvimento sustentável que sirvam de base sólida para adotar decisões em todos os níveis, e que contribuam a uma sustentabilidade auto-regulada dos sistemas integrados do meio ambiente e o desenvolvimento. (UNITED NATIONS, 1992: capítulo 40 Agenda 21).

Já os índices correspondem a um nível apurado de associação dos indicadores. Ou seja, é uma informação mais abrangente proveniente da agregação de um conjunto de indicadores ou variáveis (tratamento preliminar e parcial dos dados) capaz de interpretar a realidade de um sistema (SICHE, et al, 2007:137-148).

As maiores contribuições para a medição da sustentabilidade são obtidas junto a indicadores e índices como o Pegada Ecológica - EF (Ecological Footprint REES, 1992); o Desempenho Emergético - EMPIs (Emergy Performance Index BROWN; ULGIATI, 1997); e a Sustentabilidade Ambiental - ESI (Environmental Sustainability Index - SAMUEL-JOHNSON; ESTY, 2000). Contudo, verificam-se diversas outras proposições de indicadores e índices de sustentabilidade, mas que são pouco aplicáveis na prática, devido ao dispêndio de tempo e a necessidade de conhecimento especializado do instrumento de coleta e manipulação de dados (SICHE, et al, 2007:137-148).

Já em relação aos modelos urbanos estes permitem explorar diferentes alternativas matemáticas ou algorítmicas, das possíveis relações de causa-efeito, semelhança formal, associações, correlações, dentre outros aspectos inerentes à estrutura urbana que consiste no suporte espacial das relações sociais (ECHENIQUE, 1975: 13-16; ALEXANDER, 1980: 17). Ou seja, a modelagem urbana permite avançar nas possibilidades de análise e aplicação dos indicadores e índices 
ditos "estáticos" em predições das transformações momentâneas do cenário legal (condição futura das normativas do planejamento urbano) e cenário proposto (condição futura das alternativas de intervenção e desenho urbano em áreas novas e existentes), dispensando rotinas sistemáticas de observação direta no local (WEGENER, 1994: 18). Isto porque, modelos urbanos objetivam, sobretudo, prover um quadro simplificado e inteligivel daquilo que, fundamentalmente, são as características (físicas e conceituais) relevantes de uma situação real, no intuito de se conseguir realizar procedimentos de análise sem perder a validade dos resultados (LEE, 1973: 7; CHADWICK, 1973: 178; ECHENIQUE, 1975: 17).

\section{OS ATUAIS SISTEMAS DE CERTIFICAÇÃO: ABORDAGEM CONCEITUAL E METODOLÓGICA}

Atualmente, no Brasil, algumas certificações têm sido amplamente adotadas, como o LEED - Lidership in Energy and Environmental Design (selo greenbuilding), desenvolvido pelo Green Building Council e o AQUA - Alta Qualidade Ambiental (extraído do referencial francês HQE-Association).

O LEED é uma certificação que trabalha com uma metodologia de avaliação dos princípios ambientais e de uso de energia, por meio da obtenção de créditos distribuídos em cinco categorias de impacto ambiental e outros cinco créditos destinados à categoria de inovação e processo de projeto (PICOLI et al, 2010). No sistema de certificação LEED-ND (Neighborhood Development) para bairros e comunidades urbanas são utilizados princípios do urbanismo sustentável, os quais requerem elevados níveis de responsabilidade social e ambiental.

O Certificado AQUA - Alta Qualidade Ambiental (Fundação Carlos Alberto Vanzolini), baseado no sistema francês NF Bâtiments Tertiaires - Démarche HQE® consiste num método que aborda tanto o padrão para o sistema de gestão quanto para a qualidade ambiental da edificação (VANZOLINI, 2008), o qual foi o primeiro sistema brasileiro de certificação ambiental de edificações.

Nesta mesma linha citam-se o ASUS - que utiliza a sustentabilidade como instrumento auxiliar de projeto (ALVAREZ e SOUZA, 2011); o BREEAM - BRE 
Environmental \& Sustainability Standard, que avalia o desempenho ambiental das edificações (BREEAM, 2009); o CASBEE - Comprehensive Assessment System for Building Environmental Efficiency, que avalia desde a edificação até a cidade por meio de medidas ambientais, econômicas e sociais que implicam diretamente no desenvolvimento urbano (CASBEE, 2008); o Green Star, que pondera pesos de localização do projeto de modo a refletir as diferentes necessidades e ecologias do lugar (GREEN STAR, 2002); e, o HQE - Haute Qualité Environmentale (Guide pratique du référentiel pour la qualité environnementale des bâtiments), que utiliza multicritérios voltados à eficiência energética, o respeito ao meio ambiente e a saúde e o conforto dos ocupantes da edificação (HQE, 2014).

Constata-se que, de um modo geral, parte dos sistemas de certificação possui listas de verificação, cujos créditos são concedidos em razão de determinados critérios que consideram diferentes categorias ambientais e construtivas (PICOLLI et al, 2010; DEGANI \& CARDOSO, 2002; PINHEIRO \& SOARES, 2007). Contudo, observa-se que faltam alternativas de certificações capazes de analisar a qualidade, a eficiência e a sustentabilidade (energética, ambiental, social e econômica) de setores urbanos em cidades, principalmente junto à realidade brasileira.

\section{MÉTODO DE OBTENÇÃO DO MODELO URBANO COM BASE EM ÍNDICES E INDICADORES}

A quantidade e diversidade de variáveis existentes nos setores urbanos, associadas à significativa necessidade de readequações e alterações dimensionais dos ambientes em razão das atividades exercidas ao longo dos anos, torna complexa a tarefa de identificar e analisar estas variáveis. Segundo diferentes ritmos e temporalidades, as cidades constituem artefatos em constante processo de construção e (re)ocupação, tendendo à estabilidade e à consolidação. Possuem um pulsar de (trans)formação no qual, ao longo do tempo, partes são modificadas e outras mantidas. Contudo, determinadas variáveis socioespaciais e técnicoambientais embora não sendo permanentes, são duráveis, e se mantêm ao longo dos anos nos tecidos urbanos, tais como o sistema de vias, o perímetro das 
quadras, o parcelamento do lotes, as ocupações de áreas predominantemente residenciais, determinadas atividades não-residenciais tradicionais e/ou de grande porte, a vegetação urbana, os mananciais hídricos etc. Portanto, setores urbanos apresentam regularidades e recorrências, e essas, de acordo com o aporte conceitual e teórico adotado, podem ser mensuráveis através de índices quantitativos e qualitativos.

Como setores urbanos apresentam desempenhos variados, dependendo do aspecto que está sendo estudado, e das abordagens adotadas, faz-se necessário especificar no que consiste cada variável:

- variáveis socioespaciais: a influência da morfologia urbana em termos de forma, tamanho, disposição e quantidade de edificações, quadras e sistema de vias na dinâmica do movimento de pedestres, ciclistas e transporte público, interrelacionado com a acessibilidade e a mobilidade urbana, desde o ponto de vista do transporte, tráfego e trânsito.

- Variáveis técnico-ambientais: a adequação do setor urbano ao contexto climático, topográfico e natural do local, em termos de orientação solar, ventilação e iluminação natural, assim como a utilização de materiais disponíveis no local, pouco processados, não tóxicos, potencialmente recicláveis e culturalmente aceitos.

\subsection{MEDIDAS DE DESEMPENHO DOS SETORES URBANOS}

Considera-se que medidas de desempenho centradas na área de abrangência do setor urbano; na distribuição dos caminhos alternativos do sistema de vias; na densidade e compacidade edilícia; e, na economia de insumos naturais e antrópicos - obtidas a partir de variáveis socioespaciais e técnico-ambientais apresentam maior capacidade explicativa, cuja consideração em conjunto aprimora significativamente os parâmetros de análise das certificações atualmente utilizadas. Estas medidas de desempenho abarcam propriedades técnicas, construtivas, morfológicas, sociais, políticas, econômicas, culturais, espaciais e ambientais, as quais constituem a materialidade fundamental para a sustentabilidade em cidades. Deste modo, a expressão matemática do modelo urbano consiste num índice que 
corresponde ao sistema de pontuação baseado em dados qualitativos e quantitativos sobre um determinado setor urbano.

Obtém-se o valor do Índice de Certificação de Cidades a partir dos seguintes dados [(eq. 1); qd. 1] e expressão matemática:

\section{Quadro 1: Expressão matemática do Índice de Certificação de Cidades}

Dada a fórmula:

$I C C=$ Abrang_SU $(x$ 0,20) + Distri_CA $(x$ 0,20) + Dens_Comp $(x$ 0,20) + Ins_NA $(x \quad 0,40)$

Onde:

ICC; Índice de Certificação de Cidades

Abrang_SU; área de abrangência do setor urbano

Distri_CA; distribuição dos caminhos alternativos

Dens_Comp; densidade e compacidade

Ins_NA; insumos naturais e antrópicos

Que deve ser lida como o Índice de Certificação de Cidades é igual a ponderação da área de abrangência do setor urbano, com o somatório ponderado da distribuição dos caminhos alternativos, com o somatório ponderado da densidade e compacidade, com o somatório ponderado dos insumos naturais e antrópicos, normatizado em valores entre 0 e 1.

Fonte: original do autor (SILVA: 2015)

Na sequência são enunciadas as propriedades qualitativas e quantitativas de cada medida de desempenho que compõem as variáveis socioespaciais e técnicoambientais do Índice de Certificação de Cidades. De modo a otimizar o relato das informações, foram suprimidas as descrições das expressões matemáticas, sendo fornecida essencialmente a base conceitual de cada medida de desempenho.

\subsubsection{MEDIDA DE DESEMPENHO: ÁREA DE ABRANGÊNCIA DO SETOR URBANO}

A definição da área de abrangência do setor urbano consiste em estabelecer o limiar do recorte a partir de um determinado ponto de referência física no meio urbano. Considera-se como ponto de referência física um dado lote e/ou edificação, 
a partir do qual se determina a distância ${ }^{4}$ de passos topológicos ${ }^{5}$, de acessibilidade sintática $^{6}$, capaz de abarcar suficiente diferenciação espacial, formal e funcional. É possível verificar o quanto a área de abrangência dos setores urbanos sobrepõe áreas ambientais tais como áreas de preservação permanente, mananciais hídricos, matas ciliares, áreas de deslizamentos, áreas alagadiças, áreas de aterro sanitário, áreas de rejeitos inertes, dentre outras.

A delimitação de setores urbanos por áreas de abrangência, a partir da distância de passos topológicos de acessibilidade sintática, se justifica na medida em que possibilita definir parâmetros de comparação entre diferentes setores urbanos, viabilizando operacionalmente a etapa de levantamento de dados diretamente no local, otimizando o dispêndio de tempo com pessoal e recursos financeiros (SILVA, 2010).

\subsubsection{MEDIDA DE DESEMPENHO: DISTRIBUIÇÃO DOS CAMINHOS ALTERNATIVOS}

A partir do cálculo da acessibilidade sintática tradicional, Silva (2010) propôs a construção da medida de distribuição dos caminhos alternativos, a qual relaciona o nível de carregamento de uma linha axial somando todas as demais linhas

\footnotetext{
4 “(...) Note-se que essa 'distância' é de natureza antes topológica do que geométrica, ou seja, é obtida em razão de quantas linhas, abstraídas do sistema de espaços abertos, temos minimamente de percorrer para ir de uma dada posição, na cidade, a outra posição, e não em virtude dos metros lineares de percurso que separam minimamente essas posições. Em outras palavras, num sistema muito integrado, temos de dobrar um número pequeno de esquinas para ir, em média, de uma rua para qualquer outra do lugar. O contrário se dá num sistema menos integrado (...)" (HOLANDA, 2002: 103).

${ }^{5}$ Topologia é uma condição relacional entre partes na qual independem forma, tamanho e distância métrica, e sim da articulação entre as partes, enquanto a geometria é a descrição dos elementos físicos em relação às suas dimensões, proporções, escalas etc.

${ }^{6}$ Para medir a acessibilidade sintática por meio da integração do sistema de vias, Hillier e Hanson (1984) substituem a medida métrica pela distância topológica. A distância em Sintaxe Espacial é chamada de profundidade a qual consiste na distância topológica de um espaço a todos os demais espaços do sistema. Relações de profundidade necessariamente envolvem a noção de que o espaço aberto público pode ser acessado a partir de outros espaços adjacentes a este (HILLIER \& HANSON, 1984: 82-102).
} 
intervenientes e conectadas a esta. Isto porque, tão-somente a medida de acessibilidade sintática de uma linha axial não expressa necessariamente uma rede densa de articulação e continuidade de percursos possíveis ao longo de uma linha axial. A medida de distribuição dos caminhos alternativos (SILVA, 2010) com limitações de passos topológicos é diretamente calculada a partir do número de conexões de linhas axiais adjacentes. Estudos sintáticos têm demonstrado ${ }^{7}$ que a inteligibilidade é a propriedade-chave da estrutura espacial das cidades, responsável pelo sentido de orientação, pois possibilita o discernimento das partes, cuja configuração das áreas de estudo inteira pode ser predita pelo conhecimento da configuração ao nível local.

\subsubsection{MEDIDA DE DESEMPENHO: DENSIDADE E COMPACIDADE}

A medida de desempenho densidade e compacidade enfatiza a otimização da maior distribuição num menor distanciamento espacial entre atividades residencial e não-residencial em consonância com o movimento de pessoas, especialmente o transporte não-motorizado e o transporte público.

O princípio fundamental do adensamento urbano é o desenvolvimento compacto. Setores urbanos compactos possuem várias atividades localizadas convenientemente próximas (escolher - comparar - obter) minimizando o tempo e a energia necessários para alcançá-los e maximizando o potencial de interação. Com distâncias menores, setores urbanos compactos funcionam com infra-estrutura menos extensa e custosa (apesar de serem exigidos altos padrões de planejamento e desenho urbano) e de algum modo, preservam os ambientes naturais em relação à ocupação urbana, ao priorizar o adensamento e reaproveitamento do solo já ocupado (TOD, 2013: 24).

Em termos de desempenho, a densidade e a compacidade denotam a eficiência dos setores urbanos, na medida em que setores urbanos contínuos e regulares, compactos e densificados aproximam pessoas, intensificam as diferentes

\footnotetext{
${ }^{7}$ Para maior aprofundamento conceitual, teórico e metodológico consultar Space Syntax Symposium.
} 
atividades urbanas, otimizam os investimentos públicos, e são menos onerosos para sua manutenção. Estão associados à eficiência, por corresponder, em certa medida, a processos sociais no espaço. São economicamente sustentáveis, por fazerem uso intensivo do solo e distribuírem custos entre uma quantidade maior de usuários, dentre outras vantagens.

\subsubsection{MEDIDA DE DESEMPENHO: ECONOMIA DE INSUMOS NATURAIS E ANTRÓPICOS}

No âmbito desta pesquisa insumos consistem em cada um dos elementos (naturais e antrópicos) necessários para a vida em sociedade nas cidades. Antrópico refere-se a tudo aquilo que resulta da ação humana sobre o meio ambiente natural. Cada ação antrópica, como por exemplo, morar, trabalhar e circular terá um impacto no meio ambiente natural que é conhecido como impacto ambiental.

Esta medida de desempenho contempla procedimentos que, comprovadamente, representem economia direta ou indireta de insumos naturais e antrópicos, quais sejam:

- inovação tecnológica em fontes alternativas de energia (eólica, solar, fotovoltaica, biomassa) e eficiência dos dispositivos economizadores de água;

- redução dos incômodos e das diferentes formas de poluição: gerenciamento e seleção de lixo e resíduos inertes, tratamento adequado de esgoto, reutilização de água, programação visual e controle sonoro;

- planos, procedimentos e políticas públicas de requalificação urbana em áreas de interesse para preservação do patrimônio histórico, cultural e natural;

- $\quad$ adequação dos serviços urbanos: iluminação, limpeza e segurança pública;

- medidas operacionais voltadas ao transporte, tráfego e trânsito que visem o desenvolvimento orientado ao transporte (TOD) e Traffic calming;

- cobertura vegetal urbana em termos de forma, quantidade, distribuição e tamanho, além da preservação e plantio de espécies nativas;

- urbanidade, solidariedade e co-presença; 
- identidade, territorialidade e pertencimento;

- animação urbana, arte urbana e atividades culturais;

- a função social da propriedade no que concerne a adequação aos instrumentos do estatuto da cidade previstos em plano diretor.

\section{INSTRUMENTO PARA A TOMADA DE DECISÃO JUNTO AO PLANEJAMENTO E GESTÃO URBANA}

Transformar dados em informação, organizá-los e classificá-los, por meio de recursos computacionais, de modo a permitir a tomada de decisão em prol de algum objetivo estratégico, operacional e administrativo é o que se propôs ao vincular o Índice de Cerificação de Cidades com fundamentos da tecnologia da informação no nível do planejamento urbano de setores da cidade. Com tantos recursos disponíveis e possibilidades quase ilimitadas, os gestores públicos possuem, com a tecnologia da informação, valioso instrumento eficaz e rápido para a tomada de decisões. Entretanto, na prática raramente os sistemas de informação de cidades funcionam sistematicamente, pois são complexos, fragmentados e não respondem às necessidades imediatas.

Cidades a cada dia transformam partes de seu tecido urbano, e com isso necessitam de monitoramento constante, de modo que os regimes urbanísticos, que regem o parcelamento, o uso e a ocupação de áreas da cidade, estejam sempre atualizados e ajustados aos anseios e necessidades das pessoas. Neste sentido, a pesquisa contribui ao servir de base para ações que dizem respeito ao controle do uso do solo, estudos e relatórios de impacto ambiental e de viabilidade urbanística, para os quais são necessárias técnicas de levantamento de dados para a atribuição das possíveis causas e consequências que a urbanização de determinadas áreas pode acarretar.

A falta de instrumentos aprimorados e adequados para detectar e diagnosticar os problemas existentes e avaliar decisões alternativas na prática do planejamento urbano pode ser um impedimento à gestão urbana satisfatória. Gestores públicos possuem com a certificação, valioso instrumento eficaz e rápido para a tomada de 
decisões. Este repertório de informações a respeito do desempenho dos setores urbanos auxilia no planejamento de ações operacionais mais eficazes e condizentes com as expectativas de todos os agentes envolvidos (população, comerciantes, poder público etc), suscitando impactos verdadeiramente positivos na implementação de melhorias da qualidade de vida na cidade.

\section{CONSIDERAÇÕES FINAIS}

O desenvolvimento conjugado de índices e indicadores - tendo por base modelos urbanos para a certificação de cidades - é recente no cenário da produção de pesquisas em termos de aplicabilidade efetiva. O Índice de Certificação de Cidades contribui para ampliar o debate conceitual e metodológico em torno do desenvolvimento de novos instrumentos capazes de demonstrar diferenciações qualitativas e quantitativas da eficiência energética, com vistas à sustentabilidade social, econômica e ambiental em cidades. Ao invés de servir como meio para explicações diretas de fatos observados, o Índice de Certificação de Cidades enfoca as diferentes possibilidades de relações e interações por detrás dos acontecimentos. Fornece aos planejadores e gestores urbanos a possibilidade de adquirir conhecimento sistemático do comportamento do sistema urbano, ao simular as transformações dos setores urbanos e cogitar suas implicações socioespaciais e técnico-ambientais.

\section{REFERÊNCIAS}

ALEXANDER, C. La estructura del medio ambiente. Barcelona: Tusquets. 1980.

ECHENIQUE, M. El concepto de sistemas, modelos y teorías en los estudios urbanos. Modelos Matemáticos de la estructura espacial urbana: Aplicaciones en América Latina - Buenos Aires: Ediciones Siap, p.: 13-46, 1975.

ALVAREZ, C. E.; SOUZA, A. D. S. ASUS: Avaliação de Sustentabilidade. 2011.

Acesso em: 21 dez. 2011.

Disponível em: <http://www.lppufes.org/asus/ferramenta.php\#>

BREEAM: BRE Environmental \& Sustainability Standard. [S.I.]: BRE Global. 2009. 
BROWN, M. T.; ULGIATI, S. Emergy-based indices and ratios to evaluate sustainability: monitoring economies and technology toward environmentally sound innovation. Ecological Engineering, v. 9, n. 1-2, p. 51-69, set. 1997.

CHADWICK, G. F. Una vision sistemica del planeamiento. Barcelona: Gustavo Gilli. 1973.

CASBEE for New Construction: Comprehensive Assessment System for Building Environmental Efficiency. Institute for Building Environmental and Energy Conservation. [S.I.]. 2008

DEGANI, C. M.; CARDOSO F. F. A Sustentabilidade ao longo do ciclo de vida de edifícios: a importância da etapa de projeto arquitetônico. NUTAU 2002 - Universidade de São Paulo - Escola Politécnica. São Paulo: USP. 2002.

GREEN STAR. Green Building Council of Australia. Australia: [s.n.]. 2009.

HQE (2014). Haute Qualité Environmentale.

Acesso em: 03 out. 2014

Disponível em: <http://www.behqe.com>

HILLIER, B.; HANSON, J. The social logic of space. Cambridge: Cambridge University Press.1984

HOLANDA, F. O espaço de exceção. Brasília: EdUNB. 2002.

LEED. Lidership in Energy and Environmental Design. For New Construction and Major Renovation. Washington, U.S. Green Building Council. 2009.

LEE, C. Models in planning: an introduction to the use of quantitative models in planning. Oxford: Pergamon Press. 1973.

PICCOLI, R.; KERN, A. P.; GONZÁLEZ, M. A.; HIROTA, E. H. A certificação de desempenho ambiental de prédios: exigências usuais e novas atividades na gestão da construção. Ambiente Construído, Porto Alegre, v. 10, n. 3, p. 69-79, jul./set. 2010.

PINHEIRO, M.D. \& SOARES, L. Contributo para os mecanismos de ponderação dos critérios, no sistema Lidera, para avaliação e gestão ambiental da construção sustentável. Lisboa, 2007.

REES, W. Ecological footprints and appropriated carrying capacity: what urban economies leaves out. Environment and Urbanization, v. 4, n. 2, p. 121-130, 1992.

SAMUEL-JOHNSON, K.; ESTY, D. C. Pilot Environmental Sustainability Index Report. Davos (Switzerland): World Economic Forum: Annual Meeting, 2000. 41 p.

Acesso em: 12 out. 2014.

Disponível em: <http://sedac.ciesin.org/es/esi/ESI_00.pdf>.

SILVA, A. S. Modelagem, mensuração e simulação do movimento de pedestres e veículos. Tese (doutorado) - Universidade Federal do Rio Grande do Sul, PROPUR, Porto Alegre, RS. 2010.

SICHE, R; AGOSTINHO, F; Enrique ORTEGA, E; ROMEIRO, A. Índices versus indicadores: precisões conceituais na discussão da sustentabilidade de países. Ambiente \& Sociedade. Campinas v. X, n. 2. p. 137-148. jul.-dez. 2007 
